\section{BMJ Paediatrics Open}

\title{
Prospective observational study of universal newborn eye screening in a hospital and community setting in New Zealand
}

Samantha Kaye Simkin, ${ }^{1}$ Stuti L Misra, ${ }^{1}$ Malcolm Battin, ${ }^{2}$ Charles N J McGhee,,${ }^{1,2}$ Shuan Dai ${ }^{1,2}$

To cite: Simkin SK, Misra SL, Battin M, et al. Prospective observational study of universal newborn eye screening in a hospital and community setting in New Zealand. BMJ Paediatrics Open 2019;3:e000376. doi:10.1136/ bmjpo-2018-000376

Received 19 September 2018 Revised 17 December 2018 Accepted 19 December 2018

\section{Check for updates}

(C) Author(s) (or their employer(s)) 2019. Re-use permitted under CC BY-NC. No commercial re-use. See rights and permissions. Published by BMJ.

${ }^{1}$ Department of Ophthalmology, New Zealand National Eye Centre, Faculty of Medical and Health Sciences, University of Auckland, Auckland, New Zealand

${ }^{2}$ Auckland District Health Board, Auckland, New Zealand

Correspondence to Dr Shuan Dai; shuandai@me. com

\section{ABSTRACT}

Objective Early detection of ocular abnormalities in newborn infants is essential for timely diagnosis and treatment. This study aimed to assess wide-field digital imaging for universal newborn eye screening (UNES) to determine the prevalence of ocular abnormalities, including retinal haemorrhages, in newborn infants in New Zealand. Design Prospective ocular screening study of infants. Setting A public hospital maternity ward and a community birth centre in Auckland, New Zealand. Patients A total of 350 infants were enrolled in UNES, those with birth weight $<1250 \mathrm{~g}$ or gestational age $<30$ weeks were excluded.

Methods Wide-field digital images of the external eye and retina were captured by RetCam (Natus Medical, San Carlos, California, USA) and reviewed by an ophthalmologist via an established telemedicine methodology.

Main outcome measures Detection of ocular abnormalities, including retinal haemorrhages. Correlation between haemorrhages and maternal, obstetric and neonatal factors.

Results A total of 346 infants completed screening (median age 2 days). Retinal haemorrhages were present in 50 cases (14.5\%), two cases exhibited persistent retinal haemorrhages at 6-week follow-up. A significant increase in the odds of retinal haemorrhages was present for vaginal delivery compared with caesarean section. Other ocular abnormalities, including congenital cataract and optic nerve hypoplasia, were present in $1.4 \%$ of infants. Conclusions 0cular abnormalities were detected by UNES including congenital cataract and optic nerve hypoplasia. However, retinal haemorrhages, significantly associated with delivery modality, were the most common abnormality detected. The majority of retinal haemorrhages resolved spontaneously.

\section{INTRODUCTION}

Currently, newborn eye screening to identify ocular disorders, such as congenital cataract during the neonatal period, is predominantly performed by the red reflex test. ${ }^{1}$ Eye screening guidelines in New Zealand recommend red reflex screening in the first week of

\section{What is already known on this topic?}

Universal newborn eye screening with wide-field digital imaging in two pilot studies in developing countries reported a significant number of ocular abnormalities.

- The most common newborn ocular abnormality detected in the two reported studies by widefield digital ocular imaging is birth-related retinal haemorrhages.

- Universal newborn eye screening also detects other significant ocular abnormalities detected including cataract and retinoblastoma.

\section{What this study hopes to add?}

The presence of significant number of ocular $a b-$ normalities, including retinal haemorrhages with universal newborn eye screening, even in a high income country.

- Wide-field digital ocular imaging can be successfully performed in both community and hospital settings as part of a true universal newborn eye screening programme.

- Data on retinal haemorrhages among infants screened indicate that some retinal haemorrhages persist past 6 weeks of age, with unknown long-term consequences, but the majority resolve spontaneously.

life and at 6 weeks of age by a midwife, general practitioner or paediatrician. ${ }^{2}$ Although retinoblastoma and congenital cataracts can be detected using the red reflex test, the yield is low. ${ }^{3-5}$ Thus, potentially vision and life-threatening disorders in infants may be missed.

Infant retinal screening is standard care for all extremely premature infants at risk of retinopathy of prematurity. Retinopathy of prematurity screening in Auckland, New Zealand, is performed with wide-field digital ocular imaging which are remotely graded by an ophthalmologist. This screening method 


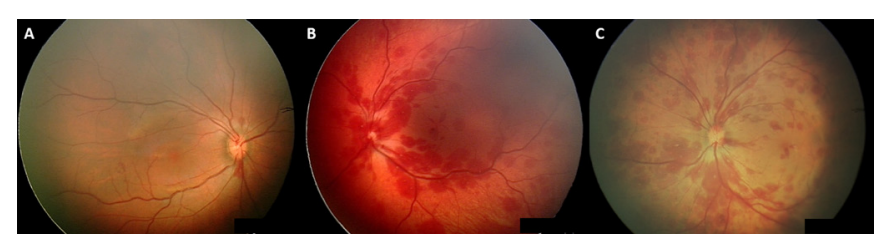

Figure 1 Wide-field digital images of retinal haemorrhages of newborn infants in UNES. (A) Isolated optic nerve haemorrhages, $(B)$ extensive haemorrhages involving the optic nerve and macula and (C) extensive, white-centred haemorrhages involving the optic nerve, macular and periphery. UNES, universal newborn eye screening.

has been incorporated into many retinopathy of prematurity screening programmes worldwide with significant incidental findings of ocular abnormalities reported. ${ }^{6-9}$

The use of wide-field digital imaging for universal newborn eye screening (UNES) to detect ocular abnormalities has been reported in both China and India. ${ }^{10} 11$ Prevalence of ocular abnormalities ranged from $4.7 \%$ to $24.4 \%$, with birth-related retinal haemorrhages $(\mathrm{RH})$ comprising over half of the abnormalities. ${ }^{1011}$ This high prevalence of RH has also been observed in the USA. ${ }^{12}$

Previous studies have been performed in selected newborn infant populations limited to hospital deliveries, with significant differences in the incidence of $\mathrm{RH}$ reported. ${ }^{10-12}$ It is unknown if these findings are specific only for infants delivered in the hospital setting or due to ethnic differences. Herein, we report the outcome of a prospective observational study of UNES with widefield digital imaging among newborn infants in both the hospital and community settings in Auckland, New Zealand.

\section{METHODS \\ Design}

UNES was designed as a prospective, observational study conducted in the Auckland region representing a diverse population of infants born in both a hospital maternity ward and community delivery centre.

\section{Patient and public involvement statement}

Patients were not directly involved in the design of this study.

\section{Examination}

Following fully informed consent, all infants born between June 2015 and December 2016 were invited to participate. Infants who were already enrolled in retinopathy of prematurity screening (infants born $<30$ weeks gestational age or $<1250 \mathrm{~g}$ birth weight) were excluded from this study. To determine the prevalence of ocular abnormalities with $95 \% \mathrm{CI}$, and absolute precision of $5 \%$, a minimum sample size of 323 infants was required. ${ }^{13}$ Therefore, the recruitment goal was 350 participating infants to account for possible cases of incomplete data, or attrition.
Wide-field digital imaging screening occurred in parallel between two locations, ADHB National Women's hospital maternity ward and BirthCare, a community birth centre. All participating infants were clinically observed by qualified midwives, paediatricians or nurses for adverse events. The entire process of image acquisition followed the well-established, local, contemporary protocol for retinopathy of prematurity screening. ${ }^{14}$

Participating infants had their eyes dilated with gutte tropicamide $1.0 \%$ (Chauvin Pharmaceuticals, Kingston-Upon-Thames, England, UK) and gutte phenylephrine $2.5 \%$ (Chauvin Pharmaceuticals, 30-60 min prior to screening. Gutte tetracaine $0.5 \%$ (Chauvin Pharmaceuticals) topical anaesthetic was instilled in each eye immediately prior to imaging. Images were captured by a nurse specialist and medical photographer using the RetCam III or RetCam Shuttle (Clarity Medical Systems, Pleasanton, California, USA).

A sterile speculum was used during screening to allow adequate ocular exposure for anterior segment and retinal imaging. Viscotears Liquid Gel (Carbomer $2 \mathrm{mg}$ /g; Norvatis Pharmaceuticals UK, Camberley, UK) was used as a coupling agent on the RetCam D130 lens between the lens and cornea. Images captured were (1) an anterior image with iris and red reflex visible, (2) retinal images including posterior pole with macula and optic nerve, temporal and nasal peripheral retina.

Encrypted, uncompressed images were stored on a secure server and were remotely reviewed by the specialist paediatric ophthalmologist, using a telemedicine model. A report of the infant's ocular findings along with the images were uploaded to the participant's electronic health record. If RH were present, the characteristics were further defined according to location and severity. Macula haemorrhages were defined as any haemorrhage within one disc diameter of the fovea, while optic nerve haemorrhages were defined as any haemorrhage involving the optic nerve head. When a haemorrhage was anterior to the retina, obscuring underlying retinal vessels, it was classified as a pre-RH. The classification of extensive RH, was used when haemorrhages numbered more than 10 and extended beyond the arcades, see figure 1 for illustration of haemorrhage types.

Infants with RH on initial screening were re-screened at 6 weeks to assess the status of $\mathrm{RH} .{ }^{15}$ Any other ocular abnormalities with potential visual or systemic impact were appropriately referred for ophthalmological or paediatric follow-up.

Data collected included gender, ethnicity, gestational age, age at screening, birth modality, birth weight, birth length, head circumference and maternal height when available from prenatal record. Maternal height was self-reported in cases where no prenatal measurement had been recorded. Maternal height was used as a surrogate for pelvic size, as pelvic dimensions are not routinely measured. ${ }^{16-18}$ 
Table 1 Demographics and characteristics of infants enrolled in UNES

\begin{tabular}{|c|c|c|}
\hline Characteristic & $\begin{array}{l}\text { Infants enrolled } \\
\text { in UNES } \\
\mathrm{N}=346 \\
\mathrm{n}(\%)\end{array}$ & $\begin{array}{l}\text { Regional newborn } \\
\text { population data } \\
2015^{19 *} \\
\mathrm{~N}=6933 \text { deliveries, } \\
\mathrm{N}=7074 \text { infants } \\
\mathrm{n}(\%)\end{array}$ \\
\hline \multicolumn{3}{|l|}{ Gender } \\
\hline Male & $177(51)$ & $3650(52)$ \\
\hline Female & $169(49)$ & $3424(48)$ \\
\hline \multicolumn{3}{|l|}{ Ethnicity } \\
\hline European & $197(57)$ & $3118(45)$ \\
\hline $\begin{array}{l}\text { Asian (including } \\
\text { Indian) }\end{array}$ & $88(25)$ & $2241(32)$ \\
\hline Māori & $27(8)$ & $469(7)$ \\
\hline Pacific peoples & $22(6)$ & 805 (12) \\
\hline Other & $12(4)$ & $300(4)$ \\
\hline \multicolumn{3}{|l|}{ Delivery modality } \\
\hline CS & $164(47) \dagger$ & $2468(36)$ \\
\hline SVD & $139(40) \dagger$ & 3594 (52) \\
\hline IVD & 43 (12) & $871(13)$ \\
\hline $\begin{array}{l}\text { GA weeks, } \\
\text { median (IQR) }\end{array}$ & $39(37.9-40.0)$ & $39(38-40)$ \\
\hline BW g, mean (SD) & 3284 (528) & 3279 (654) \\
\hline
\end{tabular}

${ }^{*}$ Regional data are reported at the maternal level for ethnicity and delivery modality.

†Delivery modality varied between newborn infants enrolled and the population of newborn infants delivered in $\mathrm{ADHB}$ in the same time period with a higher percentage of caesarean section and lower percentage of spontaneous vaginal delivery in the enrolled infants.

BW, birth wt; CS, caesarean section; GA, gestational age; IVD, instrumental vaginal delivery; SVD,

spontaneous vaginal delivery; UNES, universal newborn eye screening.

Outcome measures were (a) presence of ocular abnormalities; including RH, (b) resolution of birth-related $\mathrm{RH}$ at follow-up and (c) adverse events of screening.

\section{Statistical analysis}

Statistical analysis was completed using IBM SPSS software V.22 (Armonk). Descriptive statistics were calculated for maternal, obstetric and neonatal factors. Differences in infant characteristics were calculated by Pearson $\mathrm{X}^{2}$ test, independent sample t-test or the Mann-Whitney $\mathrm{U}$ test. Binomial logistic regression models were then used to calculate OR for birth-related RH.

\section{RESULTS}

Three hundred and fifty newborn infants were recruited for UNES. A full set of screening photos was able to be obtained for 346 (99\%) infants, 205 hospital based and

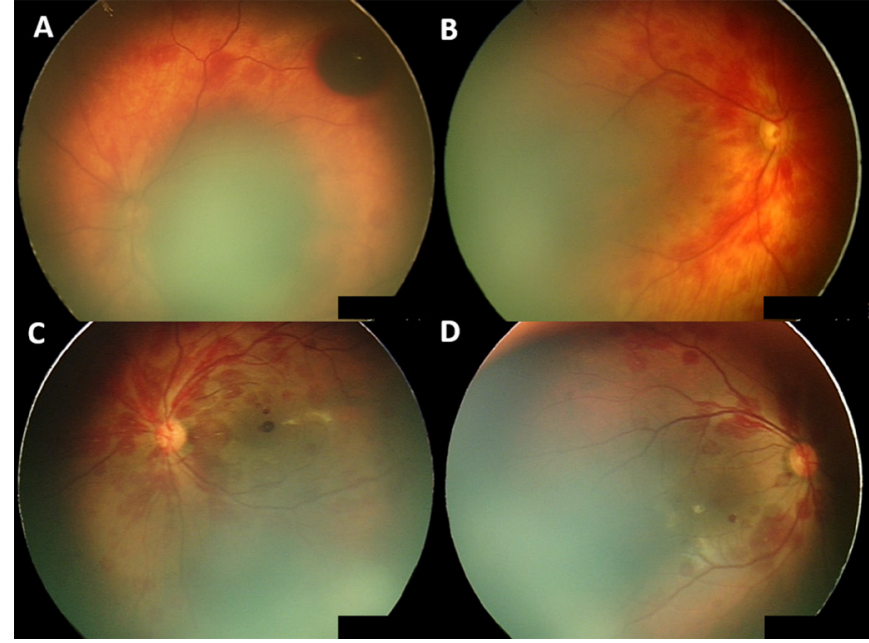

Figure 2 Initial haemorrhage presentation for two infants with non-resolving haemorrhages. Infant 1 extensive haemorrhages: $(A)$ left eye with pre-retinal haemorrhage, (B) right eye; infant 2 extensive haemorrhages, (C) left eye and (D) right eye.

141 in the community setting. Table 1 details clinical characteristics of infants enrolled in UNES, which are similar to regional infant population data reported..$^{19}$ Over half of the infants in UNES $(182 / 346)$ had vaginal delivery with $23.6 \%(43 / 182)$ of these requiring instrumental intervention including forceps or ventouse delivery, the remaining deliveries were by caesarean section. There were 11 sets of twins in the study, all were included in the analysis although outcomes of twins could potentially be linked.

The prevalence of $\mathrm{RH}$ at birth was $14.5 \%(50 / 346)$. Haemorrhages occurred bilaterally in $68 \%(34 / 50)$ of infants, unilateral haemorrhages occurred in $32 \%$ $(16 / 50)$ equally apportioned to the right and left eyes. Eight infants had RH involving both maculae. A single dense, pre-RH was noted in this study, illustrated in figure $2 \mathrm{~A}$.

Maternal, obstetric and neonatal factors were analysed independently in relation to the occurrence of $\mathrm{RH}$ (table 2). $X^{2}$-squared tests revealed significant differences in relation to the presence or absence of $\mathrm{RH}$, with respect to: delivery modality $(\mathrm{p}<0.001)$, induction of labour $(\mathrm{p}<0.001)$ and screening within 72 hours of birth $(p=0.014)$. No significant differences in ethnicity ( $p=0.602)$, or gender $(p=0.26)$ were identified. There was no significant relationship between the presence of $\mathrm{RH}$ and morphological features of the mother or child. There appeared to be an association between higher gestational age and RH $(p=0.042)$, this was not clinically significant with a median of 39.0 weeks compared with 39.4 weeks. Infants screened in the community setting were more commonly screened before 72 hours $(\mathrm{p}<0.001)$, this reflect in the percentage of haemorrhages present with $25 \%$ of infants in the community setting and $8.3 \%$ in the hospital $(\mathrm{p}<0.001)$. 
Table 2 Birth prevalence of RH by baseline characteristics in universal newborn eye screening

\begin{tabular}{|c|c|c|c|c|}
\hline Characteristic & $\begin{array}{l}\text { RH absent mean (SD) } \\
\mathrm{n}=296 \\
\mathrm{n}(\%), \text { mean } \pm \mathrm{SD}\end{array}$ & $\begin{array}{l}\text { RH present mean (SD) } \\
\mathrm{n}=50 \\
\mathrm{n}(\%) \text {, mean } \pm \mathrm{SD}\end{array}$ & $P$ value & $\begin{array}{l}\text { OR } \\
\text { (95\% Cl) }\end{array}$ \\
\hline Gender & & & 0.26 & \\
\hline Male & $155(53)$ & $22(44)$ & & \\
\hline Female & $141(48)$ & $28(56)$ & & \\
\hline Early screening & & & 0.014 & \\
\hline$<72$ hours & $218(74)$ & $45(90)$ & & 1.0 \\
\hline$\geq 72$ hours & $78(26)$ & $5(10)$ & & $0.36(0.13$ to 0.98$)$ \\
\hline Delivery modality & & & $<0.001$ & \\
\hline CS & $162(55)$ & $2(4)$ & & 1.0 \\
\hline SVD & $104(35)$ & $35(70)$ & & 26.30 (6.18 to 111.91$)$ \\
\hline IVD & $30(10)$ & $13(26)$ & & 33.61 (7.18 to 157.26$)$ \\
\hline Age at screening (days) & $3.7 \pm 4.7$ & $2.3 \pm 1.6$ & $<0.001$ & \\
\hline $\mathrm{BW}(\mathrm{g})$ & $3272 \pm 575$ & $3353 \pm 449$ & 0.35 & \\
\hline $\mathrm{HC}(\mathrm{cm})$ & $34.6 \pm 1.8$ & $34.4 \pm 1.3$ & 0.46 & \\
\hline Birth length $(\mathrm{cm})$ & $50.5 \pm 2.8$ & $50.9 \pm 1.8$ & 0.23 & \\
\hline Maternal height $(\mathrm{cm})$ & $164.9 \pm 7.1$ & $164.5 \pm 7.0$ & 0.76 & \\
\hline GA (weeks) median (IQR) & $39.0(38-40)$ & $39.4(38-40)$ & $\begin{array}{l}0.042 \\
U=6072\end{array}$ & \\
\hline
\end{tabular}

$\mathrm{RH}$, retinal haemorrhage, reported at the participant level (ie, a haemorrhage in either eye or both eyes classifies that neonate into retinal haemorrhage present group).

BW, birth wt; CS, caesarean section; GA, gestational age; HC, head circumference; IVD, instrumental vaginal delivery; SVD, spontaneous vaginal delivery.

A binomial logistic regression was performed to establish the effects of delivery modality and screening within 72 hours of age on the likelihood that RH were present. Induction was not included in the logistic regression analysis as it is closely related to delivery modality and therefore, cannot be considered an independent factor. The model demonstrated a significant increase in the odds of RH in both normal vaginal delivery (OR, 26.30; $95 \%$ CI, 6.18 to $111.91 ; \mathrm{p}<0.001)$ and instrumental vaginal delivery (OR, 33.61; 95\% CI, 7.18 to 157.26 ; $\mathrm{p}<0.001$ ) compared with infants delivered by caesarean section. Early screening, within 72 hours of birth increased the odds of RH being present $(p=0.046)$. The model also demonstrated that there was a significant decrease in the odds of RH with screening after 72 hours of age (OR, $0.36 ; 95 \%$ CI, 0.13 to $0.98 ; \mathrm{p}=0.014)$.

Follow-up was attended by $66 \%(33 / 50)$ of infants with RH, with a median follow-up time of $50 \pm 14$ days, with an IQR of 43 to 59 days. At follow-up, screening $94.0 \%$ $(31 / 33)$ of infants had complete resolution of RH. The two infants with persisting $\mathrm{RH}$ had been classified as extensive RH on initial screening following ventouse delivery, these RH took a further 3 months to resolve (see figure 2).

Other ocular abnormalities with potential visual, or systemic impact were detected in five infants (1.4\%), see figure 3. Findings included a case of congenital cataract, suspected choroidal haemangioma, congenital hypertrophy of the retinal pigment epithelium (CHRPE) and two cases of suspected optic nerve hypoplasia.

No episodes of allergic reaction, significant bradycardia or corneal abrasions were identified at or after screening. In addition, there were no reports of adverse

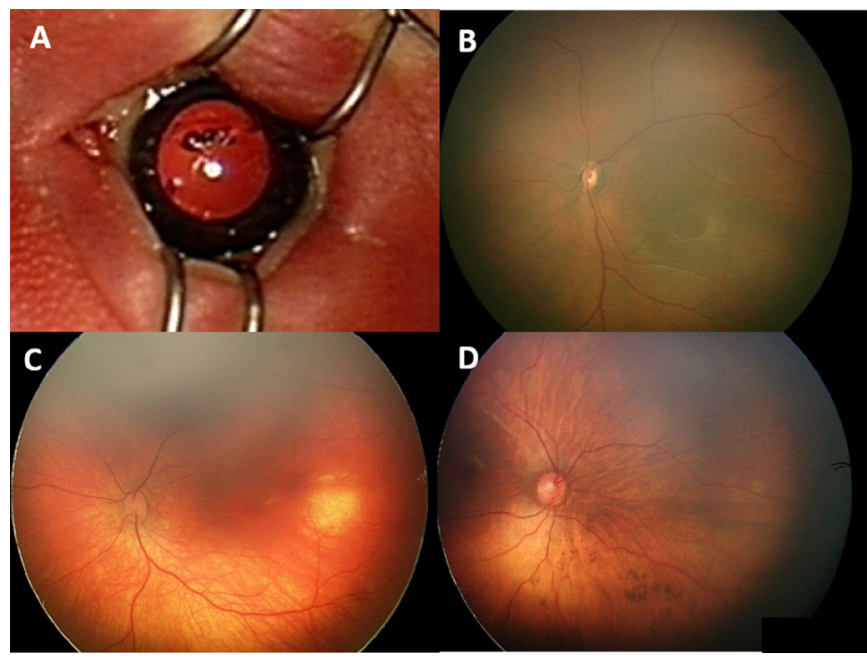

Figure 3 Ocular abnormalities detected during UNES; (A) congenital cataract, (B) optic nerve hypoplasia, (C) choroidal haemangioma and (D) inferior CHRPE. CHRPE, congenital hypertrophy of the retinal pigment epithelium; UNES, universal newborn eye screening. 
events to the screening team, throughout the period that screening was being undertaken.

\section{DISCUSSION}

This study presents the results of a prospective UNES programme in a high income country in both hospital and community settings. Other UNES programmes have been based solely in hospital settings, with the only other study in a high income country assessing RH rates rather than a complete UNES programme. ${ }^{10-12}$ UNES detected ocular abnormality in 54/346 infants who completed screening, $14.5 \%$ had $\mathrm{RH}$ and $1.4 \%$ had other ocular abnormalities, with one child having both $\mathrm{RH}$ and another ocular abnormality.

Ocular abnormality screening at birth is with the 'red reflex test' in many countries, including New Zealand. ${ }^{1}{ }^{2}$ However, this misses a significant proportion of posterior ocular abnormalities with a sensitivity of only $4 \%{ }^{.5}$ This indicates the potential need for other newborn ocular screening methods.

Detected ocular abnormalities in UNES, excluding RH were: a case each of congenital cataract, choroidal haemangioma, CHRPE and two cases of suspected optic nerve hypoplasia, one confirmed to have pituitary hormonal deficiency. Although vision may not be improved in some of the ocular abnormalities detected, such as optic nerve hypoplasia, early detection may be of benefit for other systemic health outcomes. For example, hormonal level monitoring and replacement in children with optic nerve hypoplasia, who may have septooptic dysplasia, is essential with early diagnosis key to improving outcomes. ${ }^{20}$

The prevalence of ocular abnormalities, rather than $\mathrm{RH}$, has been reported in two large studies from China and India, with a range of $2.25 \%-2.99 \% .^{10}{ }^{11}$ Systemically associated uveitis was noted in both cohorts, but no cases of ocular inflammation were noted in the our UNES study, aligning with known regional variations. ${ }^{10}{ }^{11}$ Due to the small number of each type of congenital abnormality or ocular disease in the newborn infant, large studies are required to determine the true incidence and prevalence of ocular abnormality.

A large number of RH were detected by UNES ranging from a single haemorrhage to extensive, peripheral, white-centred haemorrhages. Similar findings were reported in a smaller, hospital-based study from the USA. ${ }^{12}$ Haemorrhage presentation was most commonly bilateral $(68.7 \%)$, other studies have noted an increased rate of haemorrhages in the left eye, but no laterality difference was noted in our study. ${ }^{12}$ There was a statistically significant difference in the rate of haemorrhages present in community compared with the hospital which aligned with the significantly earlier screening occurring in the community.

We hypothesised that RH may be associated with maternal and neonatal factors including maternal height, birth weight, birth length, head circumference, gender or ethnicity. ${ }^{21}$ No significant association was detected with these factors, but mode of birth was significantly related to RH. Compared with caesarean section, the OR for RH was 26.3 normal vaginal delivery and 33.6 instrumental vaginal delivery. There is potential for imprecision in the OR calculation due to the limited number of events in the caesarean section category, hence larger studies would be beneficial. Previous reports have queried foetal head compression and thus, globe compression, as a factor in the aetiology of these haemorrhages. ${ }^{12}$ However, no relationship between maternal size relative to neonate size was noted in this or previous prospective studies of birth-related RH. ${ }^{22}$ A limitation of this study is that obstetric factors including pelvic size and duration of labour were not assessed.

The only pre-RH and persisting haemorrhages seen in this cohort occurred in newborn infants who had undergone ventouse delivery. The suction force of the vacuum extraction, has been hypothesised to cause an increase in intracranial pressure affecting central ophthalmic venous and arterial flow with retinal bleeding eventuating. ${ }^{15}$ The hypothesis that intracranial pressure changes are important in the aetiology, may explain why caesarean sections are a protective factor. ${ }^{15}$

The long-term impact of RH in newborn infants, particularly macular and prolonged haemorrhages, is currently unknown. Obscuring the visual axis during the critical period is known to cause deprivation amblyopia, ${ }^{23}$ and may also result in subtle changes in local retinal architecture. Screening before 72 hours increased the odds of RH being present, consistent with published data. ${ }^{12} 152425$ Timing of RH resolution may have potential impact on visual development and the ability to distinguish birth-related from non-accidental injury RH. A large review reported $97 \%$ of birth-related $\mathrm{RH}$ resolve by 6 weeks of age. ${ }^{15}$ The current study had a median follow-up time of just over 6 weeks with $94 \%$ of haemorrhages resolving in that timeframe, however, two cases had persisting haemorrhages at 6-week follow-up, one of which took a further 3 months to resolve. In both cases, delivered by ventouse, the RH were dense and confluent. One had a large pre-RH at initial presentation.

This prospective, screening study indicates the applicability and clinical yield of UNES in a high income country in both the hospital and community setting. Further research is required into the causative factors of birth-related RH and the long-term impact of this highly variable presentation. In addition, a full economic analysis, sensitivity and specificity calculations, as well as implementation feasibility must be determined prior to initiation of any large-scale screening.

Acknowledgements We are grateful for the support of the Save Sight Society and the University of Auckland Faculty of Medical and Health Sciences Performance Based Research Fund who funded the staff costs of the screening team and consumables for screening, respectively. This article comprised part of the PhD thesis of author Samantha Simkin, she received unrestricted funding for her $\mathrm{PhD}$ research from the University of Auckland Doctoral Scholarship and the New Zealand Association of Optometrists' Education and Research Fund. Special 
thanks to the screening team of Marie Hamoy, Steven Dakin, Holly Willing, Sarah Swanevelder and Zhen Wu.

Contributors All authors listed made substantial contributions to the conception and design of the research project. SS: acquired the data and performed the initial analysis and drafted the work. SLM, MB, CNJM, SD: all revised the work critically for intellectual content. All authors have given final approval of the version published and agree to be accountable for all aspects of the work in ensuring that questions related to the accuracy or integrity of any part of the work are appropriately investigated and resolved.

Funding The Save Sight Society, University of Auckland Faculty of Medical and Health Sciences Performance Based Research Fund, University of Auckland Doctoral Scholarship and the New Zealand Association of Optometrist's Education and Research Fund.

Competing interests None declared.

Patient consent for publication Not required.

Ethics approval This study was approved by the National Health and Disability Ethics Committee (14/NTA/183)

Provenance and peer review Not commissioned; externally peer reviewed.

Open access This is an open access article distributed in accordance with the Creative Commons Attribution Non Commercial (CC BY-NC 4.0) license, which permits others to distribute, remix, adapt, build upon this work non-commercially, and license their derivative works on different terms, provided the original work is properly cited, appropriate credit is given, any changes made indicated, and the use is non-commercial. See: http://creativecommons.org/licenses/by-nc/4.0/.

\section{REFERENCES}

1. American Academy of Pediatrics, Section on Ophthalmology, American Association for Pediatric Ophthalmology And Strabismus, et al. Red reflex examination in neonates, infants, and children. Pediatrics 2008;122:1401-4.

2. Ministry of Health. Well child/tamariki ora programme practitioner handbook: Supporting families and whanau to promote their child's health and development. Wellington: Ministry of Health, 2013

3. Rahi JS, Dezateux C. National cross sectional study of detection of congenital and infantile cataract in the United Kingdom: role of childhood screening and surveillance. The British Congenita Cataract Interest Group. BMJ 1999;318:362-5.

4. Khan AO, Al-Mesfer S. Lack of efficacy of dilated screening for retinoblastoma. J Pediatr Ophthalmol Strabismus 2005;42:205

5. Sun M, Ma A, Li F, et al. Sensitivity and specificity of red reflex test in newborn eye screening. J Pediatr 2016;179:192-6.

6. Jayadev C, Vinekar A, Bauer N, et al. Look what else we found-clinically significant abnormalities detected during routine ROP screening. Indian J Ophthalmol 2015;63:373-7.

7. Wang SK, Callaway NF, Wallenstein MB, et al. SUNDROP: six years of screening for retinopathy of prematurity with telemedicine. Can $J$ Ophthalmol 2015;50:101-6.
8. Vinekar A. IT-enabled innovation to prevent infant blindness in rural India: the KIDROP experience. Journal of Indian Business Research 2011;3:98-102

9. Dai S, Chow K, Vincent A. Efficacy of wide-field digital retinal imaging for retinopathy of prematurity screening. Clin Exp Ophthalmol 2011;39:23-9.

10. Li LH, Li N, Zhao JY, et al. Findings of perinatal ocular examination performed on 3573, healthy full-term newborns. Br J Ophthalmol 2013;97:588-91.

11. Vinekar A, Govindaraj I, Jayadev C, et al. Universal ocular screening of 1021 term infants using wide-field digital imaging in a single public hospital in India - a pilot study. Acta Ophthalmol 2015;93:e372-e376.

12. Callaway NF, Ludwig CA, Blumenkranz MS, et al. Retinal and optic nerve hemorrhages in the newborn infant: one-year results of the newborn eye screen test study. Ophthalmology 2016;123:1043-52.

13. Krejcie RV, Morgan DW. Determining sample size for research activities. Educ Psychol Meas 1970;30:607-10.

14. Kuschel C, Dai S. Retinopathy of prematurity - newborn services clinical guidelines. 2007. http://www.adhb.govt.nz/newborn/ Guidelines/Developmental/ROP.htm

15. Watts P, Maguire S, Kwok T, et al. Newborn retinal hemorrhages: a systematic review. J Aapos 2013;17:70-8.

16. Awonuga AO, Merhi Z, Awonuga MT, et al. Anthropometric measurements in the diagnosis of pelvic size: an analysis of maternal height and shoe size and computed tomography pelvimetric data. Arch Gynecol Obstet 2007;276:523-8.

17. Mahmood TA, Campbell DM, Wilson AW. Maternal height, shoe size, and outcome of labour in white primigravidas: a prospective anthropometric study. BMJ 1988;297:515-7.

18. Ridgeway B, Arias BE, Barber MD. The relationship between anthropometric measurements and the bony pelvis in African American and European American women. Int Urogynecol J 2011;22:1019-24.

19. Pot M, Sadler L. National women's annual clinical report. Auckland: Auckland District Health Board, 2015.

20. Ryabets-Lienhard A, Stewart C, Borchert M, et al. The optic nerve hypoplasia spectrum: review of the literature and clinical guidelines. Adv Pediatr 2016;63:127-46.

21. Zhao Q, Zhang $Y$, Yang $Y$, et al. Birth-related retinal hemorrhages in healthy full-term newborns and their relationship to maternal, obstetric, and neonatal risk factors. Graefes Arch Clin Exp Ophthalmol 2015;253:1021-5

22. Emerson MV, Pieramici DJ, Stoessel KM, et al. Incidence and rate of disappearance of retinal hemorrhage in newborns. Ophthalmology 2001;108:36-9

23. Solebo AL, Rahi J. Epidemiology, aetiology and management of visual impairment in children. Arch Dis Child 2014;99:375-9.

24. Hughes LA, May K, Talbot JF, et al. Incidence, distribution, and duration of birth-related retinal hemorrhages: a prospective study. $J$ Aapos 2006;10:102-6.

25. Egge K, Lyng G, Maltau JM. Retinal haemorrhages in the newborn. Acta Ophthalmol 1980;58:231-6. 